\title{
Sex Chromosome Evolution in Amniotes: Applications for Bacterial Artificial Chromosome Libraries
}

\author{
Daniel E. Janes, ${ }^{1}$ Nicole Valenzuela, ${ }^{2}$ Tariq Ezaz, ${ }^{3}$ Chris Amemiya, ${ }^{4}$ and Scott V. Edwards ${ }^{1}$ \\ ${ }^{1}$ Department of Organismic and Evolutionary Biology, Harvard University, 26 Oxford Street, Cambridge, MA 02138, USA \\ ${ }^{2}$ Department of Ecology, Evolution, and Organismal Biology, Iowa State University, 253 Bessey Hall, Ames, IA 50011, USA \\ ${ }^{3}$ Institute for Applied Ecology, University of Canberra, ACT 2601, Australia \\ ${ }^{4}$ Benaroya Research Institute at Virginia Mason, 1201 Ninth Avenue, Seattle, WA 98101-2795, USA \\ Correspondence should be addressed to Daniel E. Janes, djanes@oeb.harvard.edu
}

Received 29 July 2010; Accepted 27 September 2010

Academic Editor: Hans H. Cheng

Copyright (๑) 2011 Daniel E. Janes et al. This is an open access article distributed under the Creative Commons Attribution License, which permits unrestricted use, distribution, and reproduction in any medium, provided the original work is properly cited.

Variability among sex chromosome pairs in amniotes denotes a dynamic history. Since amniotes diverged from a common ancestor, their sex chromosome pairs and, more broadly, sex-determining mechanisms have changed reversibly and frequently. These changes have been studied and characterized through the use of many tools and experimental approaches but perhaps most effectively through applications for bacterial artificial chromosome (BAC) libraries. Individual BAC clones carry 100-200 kb of sequence from one individual of a target species that can be isolated by screening, mapped onto karyotypes, and sequenced. With these techniques, researchers have identified differences and similarities in sex chromosome content and organization across amniotes and have addressed hypotheses regarding the frequency and direction of past changes. Here, we review studies of sex chromosome evolution in amniotes and the ways in which the field of research has been affected by the advent of BAC libraries.

\section{Introduction}

Studies of the evolutionary history and functional dynamics of amniote sex chromosomes have been enabled by bacterial artificial chromosome (BAC) libraries. Here, we review several aspects of amniote sex chromosome evolution as characterized by experimentation before and after the construction of amniote BAC libraries and we suggest that the rate of discovery and reach of comparisons have been increased by BAC resources, especially for birds and reptiles in which variability of sex chromosome organization offers great opportunities for evolutionary research [1]. In addition to describing BAC-enabled research published within the last decade, we also describe experiments that will allow researchers to use BAC libraries to describe rates of evolution, dynamics of intrachromosomal variations, and frequency of independent origins of sex chromosomes.

A genomic BAC library consists of many DNA fragments representing the whole genome of an individual. Each BAC clone contains $100-200 \mathrm{~kb}$ of contiguous genomic sequences, providing probes for in situ hybridizations on chromosomes and for determining long-range organization of genes. The number of BAC clones in a library varies depending on the genome size of the species as well as the depth of the library representing the whole genome. For the construction of a BAC library, DNA is isolated and fragmented by hydroshearing, nebulization, or partial restriction digestion followed by preparative pulsed field gel fractionation. Fragments are size selected, ligated into a specialized vector, and maintained in Escherichia coli that are archived in 384-well plates at $-80^{\circ} \mathrm{C}$. Colonies representing the library are gridded onto highdensity nylon filters, and respective clone DNAs are anchored to filters by cross-linking $[2,3]$. High-density nylon filters serve as maps to BAC libraries. Radio-labeled probes can be hybridized to filters to target clones bearing sequences of interest (Figure 1). Alternatively, "smart" pooling strategies can be employed to screen BAC libraries via polymerase chain reaction [4].

Several BAC libraries and associated resources are available through the Children's Hospital Oakland Research Institute (CHORI), the Arizona Genomics Institute, the Clemson University Genomics Institute, Amplicon Express, 


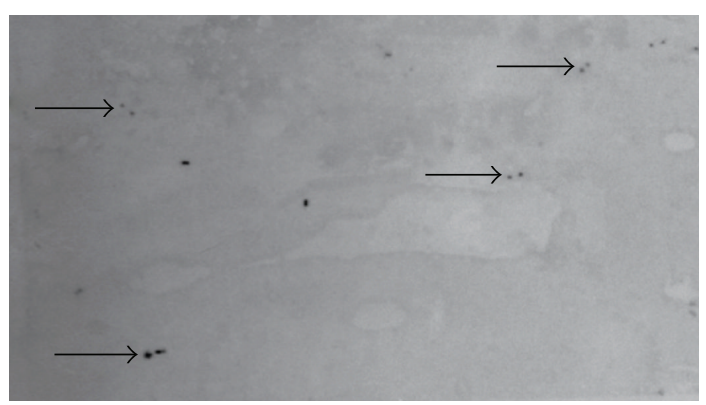

(a)

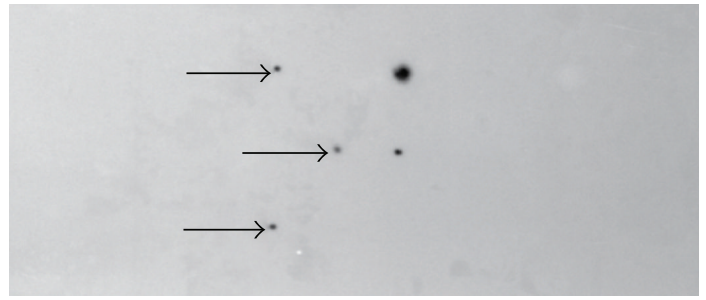

(b)

Figure 1: (a) 32P-labeled probes hybridized to sex-differentiating genes on filters representing the BAC library for painted turtle (Chrysemys picta). Two-dot signals permit the gridding of multiple 384-well plates into the same space on the filters. The two-dot pattern permits the gridding of up to 72384 -well plates on one nylon filter. Arrows indicate successful hybridization of one of several probes applied concurrently to filters. (b) Clones identified as putative positives from hybridization with a probe for Dmrt1 regridded in duplicate onto a separate filter to be hybridized with a single probe as opposed to the multiprobe approach taken to initially identify positives. The secondary hybridization permits the identification of the probe that hybridizes to each positive. Arrows indicate three hybridizations that occurred on each of two identical arrays representing only positives collected from the initial largescale hybridization. These arrays are printed side by side on the filter. Other hybridizations that were successful in only one array were rescreened to insure replicability.

Empire Genomics, and other vendors. Typically, whole plates, individual clones, or high-density nylon filters are available for purchase. The past decade has been a period of great discovery regarding amniote sex chromosomes [1, 6$9]$, and the next ten years should be even more productive as more laboratories incorporate BAC technology.

\section{A Primer on 20th Century Sex Chromosome Research}

The study of sex chromosome evolution within and beyond Amniota has a rich history that extends beyond the advent of BAC technology. Sex-specific functions of a chromosome pair in sex determination were first reported by McClung more than a century ago [10]. Since then, a series of quantitative and cytogenetic studies have generated hypotheses that still serve as pillars of contemporary experimentation [11-13]. Muller expanded on McClung's observation by suggesting that sex chromosomes evolved from an ancestral autosome pair, leading to the degeneration of the Y chromosome but not the X [14]. Haldane posited that inheritance of the degenerated sex chromosome could explain sex-specific inviability in hybrid offspring. In male heterogametic species, in which males carry $\mathrm{X}$ and $\mathrm{Y}$ sex chromosomes and females carry two $\mathrm{X}$ sex chromosomes, Haldane predicted male inviability whereas in female heterogametic species, in which females carry $Z$ and $W$ sex chromosomes and males carry two Z sex chromosomes, sex-specific inviability should affect female hybrid offspring [13]. Dobzhansky reported patterns of inheritance for sterility factors found on the X chromosome in a fly (Drosophila pseudoobscura), further suggesting that sex chromosomes are key to offspring viability but noted that such factors were also found on other chromosomes and the greater number of factors on the $\mathrm{X}$ could be attributed to differences in chromosome length rather than sex-specificity of chromosomes [15]. Three decades later, Ohno argued for a rate of sex chromosome degeneration that matches divergence times, presenting karyotypic data from snakes as evidence. W chromosomes appear to be less degenerated in characteristically ancient snakes such as colubrids that diverged from a common ancestor earlier than characteristically derived snakes like viperids that exhibit extremely degenerated W chromosomes [12]. Contemporary to Ohno's hypothesis, cytogenetic surveys rapidly improved in reporting karyotypes of many species, including amniotes $[16,17]$. In 1990, Sry, the master gene for human sex determination was identified and localized to the short (p) arm of the $\mathrm{Y}$ chromosome [18]. These and many other characterizations of the evolution and functional dynamics of sex chromosomes were enabled throughout the 20th century by integrating mating arrays, tissue culture, and chromosome staining, but molecular tools including fosmids, cosmids, and BAC libraries increased the pace and breadth of discovery.

\section{BAC-Enabled Contemporary Research on Amniote Sex Chromosomes}

Sex chromosomes house the sex-determining genes responsible for activating the developmental cascade that directs embryonic development to a male or a female fate [19]. The techniques needed to detect sex chromosomes depend in part on the evolutionary history of the sex chromosome pair. Sex chromosomes originate when a sex-determining mutation arises in a pair of autosomes $[12,20]$. This initial step is followed by the accumulation of additional mutations conferring some sex-specific advantage and by decreased recombination, sometimes involving chromosomal inversions or rearrangements $[11,12,21]$. This process may lead to the formation of two morphologically distinct sex chromosomes, exhibiting different patterns of heterochromatin accumulation and deletions, and to the degeneration of the nonrecombining heterogametic sex chromosome (Y or W) due to its higher mutation accumulation rate and ineffective selection $[12,22,23]$. In such cases, the detection of this heteromorphic pair of sex chromosomes can be carried out using classical cytogenetic techniques. For instance, a simple 
Giemsa-stained karyotype will reveal sex chromosome heteromorphisms due to differences in size while banding techniques (e.g., G-, C-, replication banding, DAPI banding, etc.) will reveal heteromorphic heterochromatin patterns.

However, sex chromosomes need not be grossly heteromorphic in size or banding pattern as the degeneration of the $\mathrm{Y}$ or $\mathrm{W}$ sex chromosome is not ubiquitous (e.g., [24] reviewed in [19]). This may be the case for sex chromosome systems at their early stages of evolution $[11,23,25-$ 28 ] or at an evolutionary stable state in some ancient sex chromosome systems (e.g., [29-31]; reviewed in [19]) Homomorphic sex chromosomes may therefore be cryptic and their detection may require molecular cytogenetic techniques such as comparative genome hybridization methods (CGH; [32]) that can reveal more subtle differences in DNA content. Recent examples of cryptic sex chromosomes in amniotes revealed by CGH include microchromosome systems in the dragon lizard (Pogona vitticeps) [33], the Chinese soft-shelled turtle (Pelodiscus sinensis) [34], and the Australian snake-necked turtle (Chelodina longicollis) [35], as well as a macrochromosome system in the Macquarie turtle (Emydura maquarii) [36]. Using CGH, differences in DNA content between the sexes are revealed by the differential hybridization pattern on chromosomal spreads of male and female genomic DNA previously labeled with two different fluorochromes.

In the past ten years, mapping of sex chromosomes has continued apace of overall genome research, enabled, in part, by fluorescent in situ hybridization (FISH) of cDNAs, cosmids, and BACs. BAC-FISH uses DNA probes derived from BAC libraries for the detection of sequences in target chromosomes [37, 38]. This technique can be used to determine the chromosomal location of specific genes (gene mapping) and to detect rearranged regions in chromosomes (breakpoints) [39, 40] where genes have changed order or location. Thus, when applied in a phylogenetic and comparative framework, BAC-FISH can be used to test hypotheses about the evolutionary history of chromosomes [39]. Recent studies in this area exemplify the utility of applying this technique to our understanding of sex chromosome evolution in amniotes [5, 41-45]. For example, gDNA-FISH permitted the localization of Dmrt1 to the $\mathrm{Z}$ but not the W chromosome in emu (Dromaius novaehollandiae) [46]. In emu, Dmrt1 is the only known marker identified to date that is solely Z linked. Beyond Dmrt1, emu sex chromosomes appear to consist entirely of the shared pseudoautosomal region (PAR) [47]. The singular Z-linkage of Dmrt1 in emu lends additional support to the report that Dmrt1 is the sexdetermining gene in birds, akin to Sry in most mammals [48]. Janes et al. [47] mapped random and targeted BACs to either autosomes or the PAR of sex chromosomes in emu. End-sequences of mapped BACs demonstrated higher rates of recombination in the PAR than on autosomes, despite equivalent population sizes of pseudoautosomal and autosomal loci in wild emu populations, assuming balanced sex ratios. Also, cDNA clones have been mapped to sex chromosomes of the Japanese four-striped rat snake (Elaphe quadrivirgata), Burmese python (Python molurus bivittatus), the habu (Trimeresurus flavoviridis), a species of gecko
(Gekko hokouensis) [49], and the Chinese soft-shelled turtle (Pelodiscus sinensis) [50], demonstrating a lack of homology of $\mathrm{Z}$ and $\mathrm{W}$ chromosomes among snakes, birds, and turtles, indicating independent origins of sex chromosomes in these clades [51]. However, homology between chicken and a gecko over six markers suggests the possibility of shared ancestry.

Bacterial artificial chromosomes are also frequently used as fingerprint maps to assign sequences to chromosomes and organize linkage groups for the improvement of genome assemblies [52]. BACs are particularly useful for sequencing fractions of genomes, as in the assembly of the malespecific region of the $\mathrm{Y}$ chromosomes in human (Homo sapiens) and chimpanzee (Pan troglodytes) [53]. Comparative physical mapping of sex chromosomes has employed BACs for species including but not limited to cow (Bos taurus) [54], domestic cat (Felis catus) [55], black muntjac (Muntiacus crinifrons) [56, 57], elephant (Loxodonta africana) [58], horse (Equus caballus) [59], South American opossum (Monodelphis domestica) $[60,61]$, tammar wallaby (Macropus eugenii) $[60,62,63]$, short-beaked echidna (Tachyglossus aculeatus) $[64,65]$, platypus (Ornithorhynchus anatinus) [63-68], and chicken (Gallus gallus) [69-71]. Mapping of degenerated sex chromosomes, in particular, is aided by BACs because of the low gene and high repeat content that are typical of degenerated sex chromosomes. These characteristics can complicate shotgun sequencing and linkage map analyses.

Contemporary research on the evolutionary history of amniote sex chromosomes is concerned with the causes and frequency of origination of sex chromosomes from ancestral autosomes and the implications of subsequent chromosomal degeneration [72]. Comparison of sex chromosomes within and among species has benefited enormously from the availability of BAC libraries, because they provide researchers the opportunity to find the genomic position of long DNA sequences that contain a targeted marker of phenotypic interest (such as a disease-causing locus) or represent a landmark genomic location for comparative purposes. Once a BAC clone that represents a marker or map location has been identified, then cross-species FISH allows researchers to reconstruct evolutionary history. For example, BAC clones from the library for the Australian dragon lizard that were known to contain genes that mapped to sex chromosomes of either snake or chicken were FISH mapped to dragon lizard chromosome spreads, demonstrating a lack of homology of either snake or chicken sex-linked genes to sequence on the sex chromosomes of dragon lizards [5]. Interestingly, while chicken sex chromosomes are homologous to chromosomes 2 in both snakes and dragon lizards, snake sex chromosomes were found to be homologous to chromosome 6 in dragon lizards, suggesting independent evolution of ZW sex chromosomes in squamate reptiles (Figure 2; $[5,51]$ ).

\section{Future Directions: Potential Applications of BAC Libraries}

Several strategies can be used to identify sex-linked markers in species with heteromorphic sex chromosomes. For 


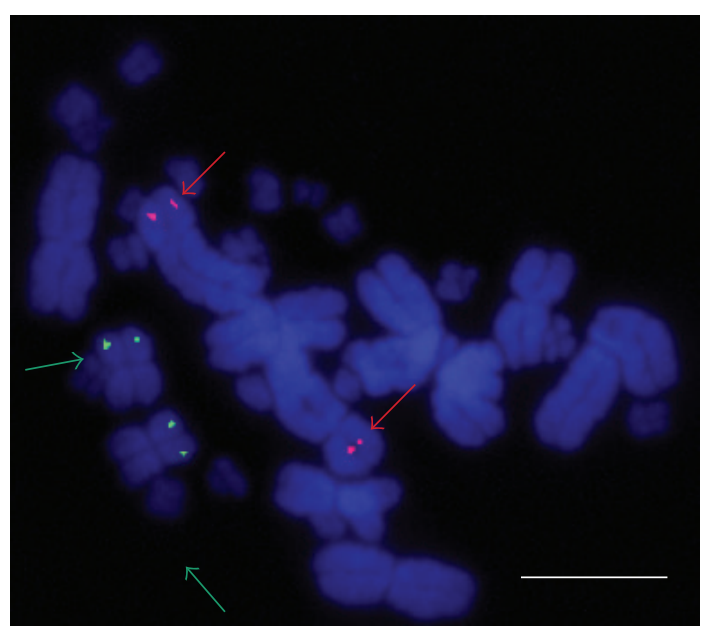

FIGURE 2: An example of two-color BAC-FISH showing hybridizations of BAC clones containing sex-linked genes from snake and chicken in a metaphase chromosome spread from an Australian bearded dragon (Pogona vitticeps). Red arrows indicate hybridization signals of a BAC clone containing chicken sex-linked gene CHD1 (red signals) onto the short arms of chromosomes 2 in P. vitticeps; green arrows indicate hybridization signals of a BAC clone containing snake sexlinked gene KLF6 onto the short arms of chromosomes 6 in $P$. vitticeps, thus showing nonhomology of ZZ/ZW sex chromosomes in reptiles [5]. Scale bar indicates $10 \mu \mathrm{m}$.

organisms with a sequenced genome, targeted BAC library screening can be performed to generate sex-chromosomespecific probes [73]. For nonmodel organisms lacking a sequenced genome, several approaches are possible. First, a large number of random BAC clones could be FISH mapped onto chromosomal spreads of the target species to determine which clones map to the sex chromosomes. The total number of hybridized BAC clones depends on the coverage of the BAC library and the diploid number of target species, and it should be in large enough numbers to ensure that at least a few clones hybridize to the sex chromosome pair. While this is a time-consuming and probabilistic approach, it may be useful for some target nonmodel species. A second approach involves the capture of the target sex chromosomes by microdissection or flow sorting, which can then be amplified to generate probes for BAC library screening (e.g., $[62,74])$. For nonmodel organisms that also lack a BAC library, a BAC library from another closely related species could be used for screening. An additional approach to detect sex-linked genes in a nonmodel taxon is to screen for $\mathrm{BAC}$ clones containing genes known to be linked to sex chromosomes in other amniotes. Sex-linked BAC-clone probes generated by any of these approaches can then be employed for physical mapping in candidate species as described above to identify their location and synteny. Such mapping data can then be used in subsequent construction of a comparative genomic map among amniotes to test evolutionary hypotheses on the origin and evolution of sex chromosomes.

\section{Acknowledgments}

The authors thank Joel Sweetser, Samuel Pokross, Chad Kohler, and Katherine Falb for their thoughtful reviews of this manuscript. Funding for this paper and for continued experimentation to characterize sex chromosome evolution in turtles through the use of BAC libraries was provided by NSF Grant \# MCB0817687 to N. Valenzuela and S. V. Edwards. D. E. Janes was also supported by NIH NRSA Grant \# 1F32GM072494. The construction of BAC libraries for several amniote species was supported by the NSF Grant no. 0431717 to S. V. Edwards, C. Amemiya, and J. Robert Macey. T. Ezaz was partially supported by the Discovery Project Grant \#DP0881196 awarded by the Australian Research Council to Stephen Sarre, Arthur Georges, and S. V. Edwards. Also, they thank Dr. Engy Edward for inviting this paper as a contribution to the Journal of Biomedicine and Biotechnology.

\section{References}

[1] D. E. Janes, C. L. Organ, M. K. Fujita et al., "Genome evolution in Reptilia, the sister group of mammals," Annual Review of Genomics and Human Genetics, vol. 239, pp. 239-264, 2010.

[2] A. M. Shedlock, D. E. Janes, and S. V. Edwards, "Amniote phylogenomics: testing evolutionary hypotheses with BAC library scanning and targeted clone analysis of large-scale DNA sequences from reptiles," in Phylogenomics, W. Murphy, Ed., Humana Press, Totowa, NJ, USA, 2008.

[3] M. T. Ross, S. LaBrie, J. McPherson et al., "Screening largeinsert libraries by hybridization," in Current Protocols in Human Genetics, A. Boyl, Ed., pp. 5.6.1-5.6.52, New York, NY, USA, John Wiley \& Sons, 1999.

[4] Y.-S. Yim, P. Moak, H. Sanchez-Villeda et al., "A BAC pooling strategy combined with PCR-based screenings in a large, highly repetitive genome enables integration of the maize genetic and physical maps," BMC Genomics, vol. 8, article 47, 2007.

[5] T. Ezaz, B. Moritz, P. Waters, J. A. Marshall Graves, A. Georges, and S. D. Sarre, "The ZW sex microchromosomes of an Australian dragon lizard share no homology with those of other reptiles or birds," Chromosome Research, vol. 17, pp. 965-973, 2009.

[6] R. Stiglec, T. Ezaz, and J. A. M. Graves, "A new look at the evolution of avian sex chromosomes," Cytogenetic and Genome Research, vol. 117, no. 1-4, pp. 103-109, 2007.

[7] T. Ezaz, R. Stiglec, F. Veyrunes, and J. A. Marshall Graves, "Relationships between Vertebrate ZW and XY Sex Chromosome Systems," Current Biology, vol. 16, no. 17, pp. R736R743, 2006.

[8] J. E. Mank, "The W, X, Y and Z of sex-chromosome dosage compensation," Trends in Genetics, vol. 25, no. 5, pp. 226-233, 2009.

[9] J. E. Mank, "Sex chromosomes and the evolution of sexual dimorphism: lessons from the genome," American Naturalist, vol. 173, no. 2, pp. 141-150, 2009.

[10] C. E. McClung, "The accessory chromosome-sex determinant," Biological Bulletin, vol. 3, pp. 43-84, 1902.

[11] J. J. Bull, Evolution of Sex Determining Mechanisms, Benjamin/Cummings, Menlo Park, Calif, USA, 1983.

[12] S. Ohno, Sex Chromosomes and Sex-Linked Genes, Springer, Berlin, Germany, 1967. 
[13] J. B. S. Haldane, "Sex ratio and unisexual sterility in hybrid animals," Journal of Genetics, vol. 12, pp. 101-109, 1922.

[14] H. J. Muller, "A gene for the fourth chromosome of Drosophila," Journal of Experimental Zoology, vol. 17, no. 325, p. 336, 1914.

[15] T. Dobzhansky, "Studies on hybrid sterility. II. Localization of sterility factors in Drosophila pseudoobscura hybrids," Genetics, vol. 21, pp. 113-135, 1936.

[16] T. C. Hsu and K. Benirschke, An Atlas of Mammalian Chromosomes, Springer, Berlin, Germany, 1977.

[17] K. Benirschke, T. C. Hsu, and F. L. Roberts, Chromosome Atlas: Fish, Amphibians, Reptiles, and Birds, Springer, Berlin, Germany, 1971.

[18] A. H. Sinclair, P. Berta, M. S. Palmer et al., "A gene from the human sex-determining region encodes a protein with homology to a conserved DNA-binding motif," Nature, vol. 346, no. 6281, pp. 240-244, 1990.

[19] N. Valenzuela, "Co-evolution of genomic structure and selective forces underlying sexual development and reproduction," Cytogenetic and Genome Research, vol. 127, no. 2-4, pp. 232 241,2010

[20] B. Charlesworth, "The evolution of sex chromosomes," Science, vol. 251, no. 4997, pp. 1030-1033, 1991.

[21] W. S. Modi and D. Crews, "Sex chromosomes and sex determination in reptiles: commentary," Current Opinion in Genetics and Development, vol. 15, no. 6, pp. 660-665, 2005.

[22] P. D. Waters, M. C. Wallis, and J. A. M. Graves, "Mammalian sex-Origin and evolution of the Y chromosome and SRY," Seminars in Cell and Developmental Biology, vol. 18, no. 3, pp. 389-400, 2007.

[23] E. J. Vallender and B. T. Lahn, "How mammalian sex chromosomes acquired their peculiar gene content," BioEssays, vol. 26, no. 2, pp. 159-169, 2004.

[24] J. W. Sites, J. W. Bickham, and M. W. Haiduk, "Banded karyotypes of 6 taxa of kinosternid turtles," Copeia, no. 4, pp. 692-698, 1979.

[25] R. Gorelick, "Theory for why dioecious plants have equal length sex chromosomes," American Journal of Botany, vol. 92, no. 6, pp. 979-984, 2005.

[26] Z. Liu, P. H. Moore, H. Ma et al., "A primitive Y chromosome in papaya marks incipient sex chromosome evolution," Nature, vol. 427, no. 6972, pp. 348-352, 2004.

[27] J.-N. Volff and M. Schartl, "Variability of genetic sex determination in poeciliid fishes," Genetica, vol. 111, no. 1-3, pp. 101110, 2001.

[28] A. J. Solari, Sex Chromosomes and Sex Determination in Vertebrates, CRC Press, Baco Raton, Fla, USA, 1994.

[29] J. E. Mank and H. Ellegren, "Parallel divergence and degradation of the avian W sex chromosome," Trends in Ecology and Evolution, vol. 22, no. 8, pp. 389-391, 2007.

[30] M. I. Pigozzi and A. J. Solari, "The ZW pairs of two paleognath birds from two orders show transitional stages of sex chromosome differentiation," Chromosome Research, vol. 7, no. 7, pp. 541-551, 1999.

[31] M. Nakamura, "Sex determination in amphibians," Seminars in Cell and Developmental Biology, vol. 20, no. 3, pp. 271-282, 2009.

[32] A. Kallioniemi, O.-P. Kallioniemi, D. Sudar et al., "Comparative genomic hybridization for molecular cytogenetic analysis of solid tumors," Science, vol. 258, no. 5083, pp. 818-821, 1992.

[33] T. Ezaz, A. E. Quinn, I. Miura, S. D. Sarre, A. Georges, and J. A. Marshall Graves, "The dragon lizard Pogona vitticeps has ZZ/ZW micro-sex chromosomes," Chromosome Research, vol. 13, no. 8, pp. 763-776, 2005.
[34] A. Kawai, C. Nishida-Umehara, J. Ishijima, Y. Tsuda, H. Ota, and Y. Matsuda, "Different origins of bird and reptile sex chromosomes inferred from comparative mapping of chicken Z-linked genes," Cytogenetic and Genome Research, vol. 117, no. 1-4, pp. 92-102, 2007.

[35] T. Ezaz, N. Valenzuela, F. Grützner et al., "An XX/XY sex microchromosome system in a freshwater turtle, Chelodina longicollis (Testudines: Chelidae) with genetic sex determination," Chromosome Research, vol. 14, no. 2, pp. 139-150, 2006.

[36] P. A. Martinez, T. Ezaz, N. Valenzuela, A. Georges, and J. A. Marshall Graves, "An XX/XY heteromorphic sex chromosome system in the Australian chelid turtle Emydura macquarii: a new piece in the puzzle of sex chromosome evolution in turtles," Chromosome Research, vol. 16, no. 6, pp. 815-825, 2008.

[37] T. Liehr, A. Weise, A. Heller et al., "Multicolor chromosome banding (MCB) with YAC/BAC-based probes and regionspecific microdissection DNA libraries," Cytogenetic and Genome Research, vol. 97, no. 1-2, pp. 43-50, 2002.

[38] P. R. Langer, A. A. Waldrop, and D. C. Ward, "Enzymatic synthesis of biotin-labeled polynucleotides-novel nucleic acid affinity probes," Proceedings of the National Academy of Sciences of the United States of America, vol. 78, no. 11, pp. 6633-6637, 1981.

[39] A. Weise, H. Starke, K. Mrasek, U. Claussen, and T. Liehr, "New insights into the evolution of chromosome 1," Cytogenetic and Genome Research, vol. 108, no. 1-3, pp. 217-222, 2005.

[40] D. Gisselsson, E. Pålsson, M. Höglund et al., "Differentially amplified chromosome 12 sequences in low- and high-grade osteosarcoma," Genes Chromosomes and Cancer, vol. 33, no. 2, pp. 133-140, 2002.

[41] P. J. Das, B. P. Chowdhary, and T. Raudsepp, "Characterization of the bovine pseudoautosomal region and comparison with sheep, goat, and other mammalian pseudoautosomal regions," Cytogenetic and Genome Research, vol. 126, no. 1-2, pp. 139147, 2009.

[42] T. Ezaz, A. E. Quinn, S. D. Sarre, D. O’Meally, A. Georges, and J. A. Marshall Graves, "Molecular marker suggests rapid changes of sex-determining mechanisms in Australian dragon lizards," Chromosome Research, vol. 17, no. 1, pp. 91-98, 2009.

[43] Y. Itoh, K. Kampf, and A. P. Arnold, "Comparison of the chicken and zebra finch $\mathrm{Z}$ chromosomes shows evolutionary rearrangements," Chromosome Research, vol. 14, no. 8, pp. 805-815, 2006.

[44] I. A. Ferreira and C. Martins, "Physical chromosome mapping of repetitive DNA sequences in Nile tilapia Oreochromis niloticus: evidences for a differential distribution of repetitive elements in the sex chromosomes," Micron, vol. 39, no. 4, pp. 411-418, 2008.

[45] R. B. Phillips, J. J. Dekoning, A. B. Ventura et al., "Recombination is suppressed over a large region of the rainbow trout y chromosome," Animal Genetics, vol. 40, no. 6, pp. 925-932, 2009.

[46] S. Shetty, P. Kirby, D. Zarkower, and J. A. M. Graves, "DMRT1 in a ratite bird: evidence for a role in sex determination and discovery of a putative regulatory element," Cytogenetic and Genome Research, vol. 99, no. 1-4, pp. 245-251, 2002.

[47] D. E. Janes, T. Ezaz, J. A. M. Graves, and S. V. Edwards, "Recombination and nucleotide diversity in the sex chromosomal pseudoautosomal region of the emu, Dromaius novaehollandiae," Journal of Heredity, vol. 100, no. 2, pp. 125$136,2009$. 
[48] C. A. Smith, K. N. Roeszler, T. Ohnesorg et al., "The avian Zlinked gene DMRT1 is required for male sex determination in the chicken," Nature, vol. 461, no. 7261, pp. 267-271, 2009.

[49] A. Kawai, J. Ishijima, C. Nishida et al., "The ZW sex chromosomes of Gekko hokouensis (Gekkonidae, Squamata) represent highly conserved homology with those of avian species," Chromosoma, vol. 118, no. 1, pp. 43-51, 2009.

[50] T. Kawagoshi, Y. Uno, K. Matsubara, Y. Matsuda, and C. Nishida, "The ZW micro-sex chromosomes of the chinese soft-shelled turtle (Pelodiscus sinensis, trionychidae, testudines) have the same origin as chicken chromosome 15," Cytogenetic and Genome Research, vol. 125, no. 2, pp. 125-131, 2009.

[51] K. Matsubara, H. Tarui, M. Toriba et al., "Evidence for different origin of sex chromosomes in snakes, birds, and mammals and step-wise differentiation of snake sex chromosomes," Proceedings of the National Academy of Sciences of the United States of America, vol. 103, no. 48, pp. 18190-18195, 2006.

[52] W. C. Warren, D. F. Clayton, H. Ellegren et al., "The genome of a songbird," Nature, vol. 464, no. 7289, pp. 757-762, 2010.

[53] J. F. Hughes, H. Skaletsky, T. Pyntikova et al., "Chimpanzee and human y chromosomes are remarkably divergent in structure and gene content," Nature, vol. 463, no. 7280, pp. 536-539, 2010.

[54] A.-S. van Laere, W. Coppieters, and M. Georges, "Characterization of the bovine pseudoautosomal boundary: documenting the evolutionary history of mammalian sex chromosomes," Genome Research, vol. 18, no. 12, pp. 1884-1895, 2008.

[55] A. J. P. Wilkerson, T. Raudsepp, T. Graves et al., "Gene discovery and comparative analysis of $\mathrm{X}$-degenerate genes from the domestic cat Y chromosome," Genomics, vol. 92, no. 5, pp. 329-338, 2008.

[56] L. Huang, J. Chi, J. Wang, W. Nie, W. Su, and F. Yang, "Highdensity comparative BAC mapping in the black muntjac (Muntiacus crinifrons): molecular cytogenetic dissection of the origin of MCR 1p+4 in the X1X2Y1Y2Y3 sex chromosome system," Genomics, vol. 87, no. 5, pp. 608-615, 2006.

[57] Q. Zhou, J. Wang, L. Huang et al., "Neo-sex chromosomes in the black muntjac recapitulate incipient evolution of mammalian sex chromosomes," Genome Biology, vol. 9, no. 6, article R98, 2008.

[58] C. L. R. Delgado, P. D. Waters, C. Gilbert, T. J. Robinson, and J. A. M. Graves, "Physical mapping of the elephant X chromosome: conservation of gene order over 105 million years," Chromosome Research, vol. 17, pp. 917-926, 2009.

[59] T. Raudsepp and B. P. Chowdhary, "The horse pseudoautosomal region (PAR): characterization and comparison with the human, chimp and mouse PARs," Cytogenetic and Genome Research, vol. 121, no. 2, pp. 102-109, 2008.

[60] J. E. Deakin, E. Koina, P. D. Waters et al., "Physical map of two tammar wallaby chromosomes: a strategy for mapping in non-model mammals," Chromosome Research, vol. 16, no. 8, pp. 1159-1175, 2008.

[61] L. S. Davidow, M. Breen, S. E. Duke, P. B. Samollow, J. R. McCarrey, and J. T. Lee, "The search for a marsupial XIC reveals a break with vertebrate synteny," Chromosome Research, vol. 15, no. 2, pp. 137-146, 2007.

[62] N. Sankovic, M. L. Delbridge, F. Grützner, M. A. FergusonSmith, P. C. M. O'Brien, and J. A. Marshall Graves, "Construction of a highly enriched marsupial Y chromosome-specific BAC sub-library using isolated Y chromosomes," Chromosome Research, vol. 14, no. 6, pp. 657-664, 2006.
[63] C. A. Edwards, W. Rens, O. Clarke et al., "The evolution of imprinting: chromosomal mapping of orthologues of mammalian imprinted domains in monotreme and marsupial mammals," BMC Evolutionary Biology, vol. 7, article 157, 2007.

[64] W. Rens, P. C. M. O’Brien, F. Grützner et al., “The multiple sex chromosomes of platypus and echidna are not completely identical and several share homology with the avian Z," Genome Biology, vol. 8, no. 11, article R243, 2007.

[65] J. C. Dohm, E. Tsend-Ayush, R. Reinhardt, F. Grützner, and H. Himmelbauer, "Disruption and pseudoautosomal localization of the major histocompatibility complex in monotremes," Genome Biology, vol. 8, no. 8, article no. R175, 2007.

[66] F. Grützner, W. Rens, E. Tsend-Ayush et al., "In the platypus a meiotic chain of ten sex chromosomes shares genes with the bird Z and mammal X chromosomes," Nature, vol. 432, no. 7019, pp. 913-917, 2004.

[67] D. McMillan, P. Miethke, A. E. Alsop et al., "Characterizing the chromosomes of the platypus (Ornithorhynchus anatinus)," Chromosome Research, vol. 15, no. 8, pp. 961-974, 2007.

[68] F. Veyrunes, P. D. Waters, P. Miethke et al., "Bird-like sex chromosomes of platypus imply recent origin of mammal sex chromosomes," Genome Research, vol. 18, no. 6, pp. 965-973, 2008.

[69] D. W. Bellott, H. Skaletsky, T. Pyntikova et al., "Convergent evolution of chicken $\mathrm{Z}$ and human $\mathrm{X}$ chromosomes by expansion and gene acquisition," Nature, vol. 466, no. 7306, pp. 612-616, 2010.

[70] K. Nam and H. Ellegren, "The chicken (Gallus gallus) Z chromosome contains at least three nonlinear evolutionary strata," Genetics, vol. 180, no. 2, pp. 1131-1136, 2008.

[71] C. Chapus and S. V. Edwards, "Genome evolution in Reptilia: in silico chicken mapping of 12,000 BAC-end sequences from two reptiles and a basal bird," BMC Genomics, vol. 10, no. 2, article S8, 2009.

[72] D. E. Janes, C. L. Organ, and S. V. Edwards, "Variability in sex-determining mechanisms influences genome complexity in reptilia," Cytogenetic and Genome Research, vol. 127, no. 24, pp. 242-248, 2010.

[73] N. P. Bohne, C. Schultheis, Q. Zhou et al., "Identification of new gene candidates on the sex chromosomes of the platyfish Xiphophorus maculatus," Cybium, vol. 32, pp. 69-71, 2008.

[74] R. Zimmer, W. A. King, and A. M. V. Gibbins, "Generation of chicken Z-chromosome painting probes by microdissection for screening large-insert genomic libraries," Cytogenetics and Cell Genetics, vol. 78, no. 2, pp. 124-130, 1997. 

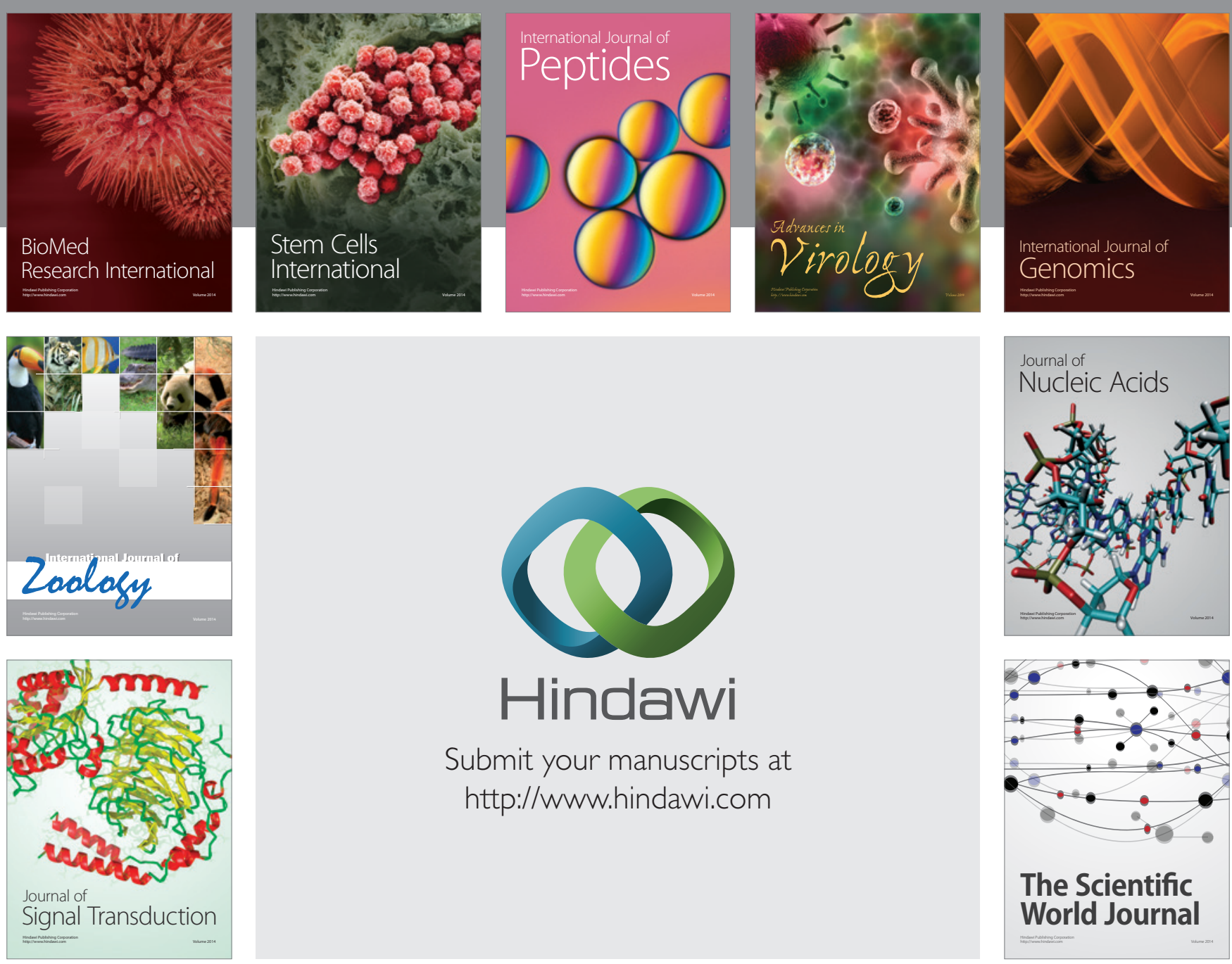

Submit your manuscripts at

http://www.hindawi.com
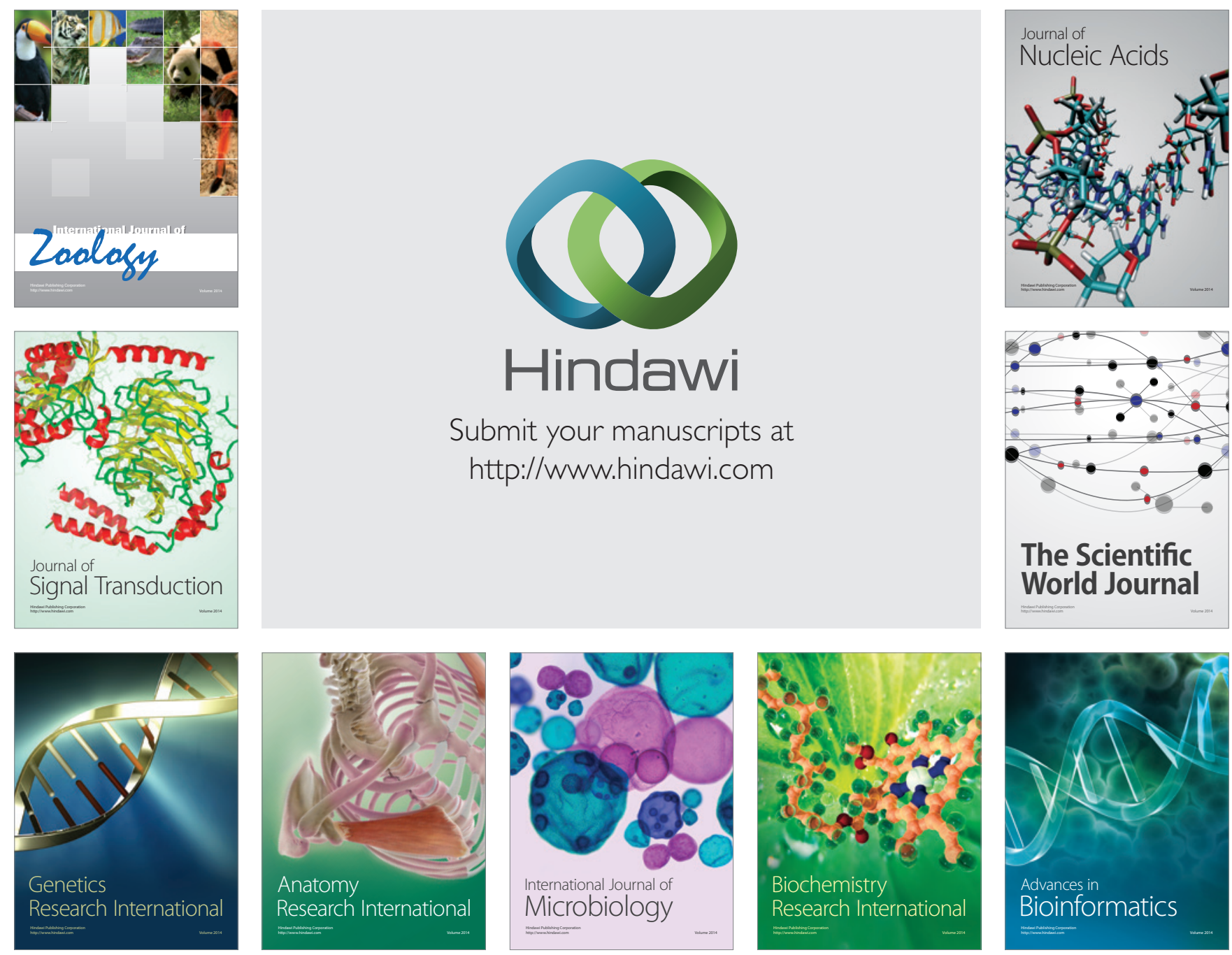

The Scientific World Journal
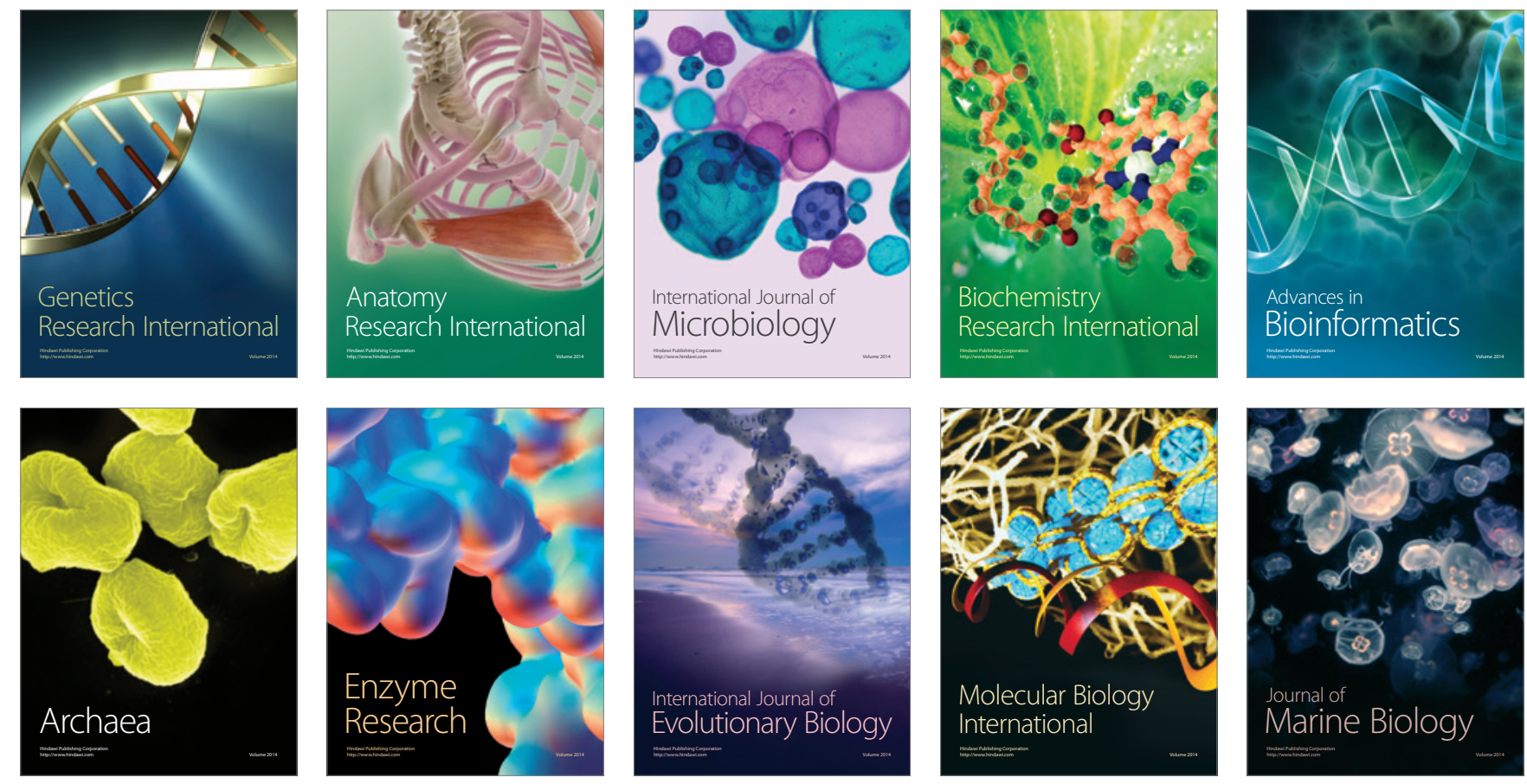